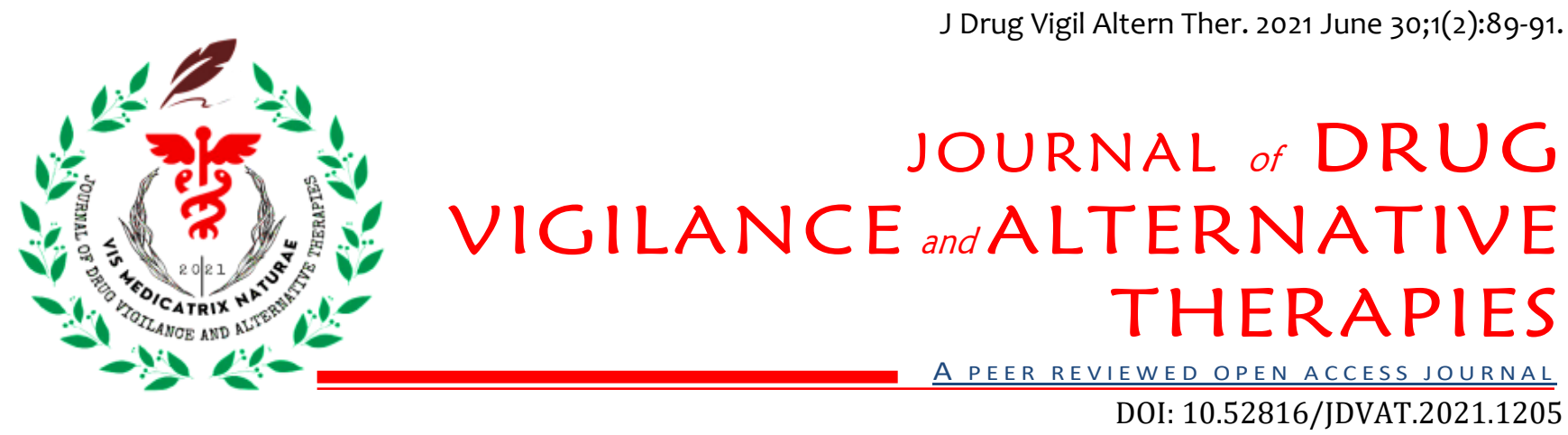

\title{
Pharmacovigilance Education for Dental Students
}

Mohammed Khalaid, Maimoona Khatoon

Department of Public Health Dentistry, MNR Dental College \& Hospital, Dr. N. T. R. University of Health Sciences, Sanga Reddy District, Telangana, India - 502294.

Reach the author at mohammed.khalaid@gmail.com

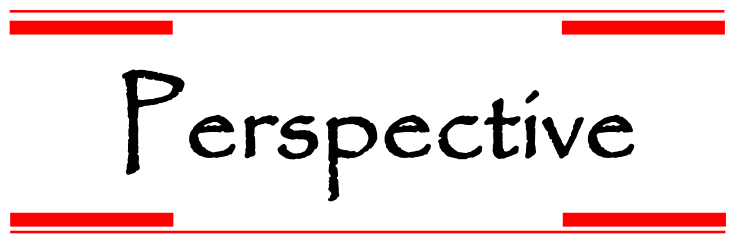

$\mathrm{T}$ he primary purpose of everyone associated in treating patients and prescribing medications is to ensure that medicines are administered safely. The safe use of drugs in dentistry is critical to the patient's well-being. The dental discipline is involved in the use of a variety of drugs in the treatment of oral diseases. The disclosure of ADR of bisphosphonate-associated osteonecrosis of the jaws alerted the dental community in 2005. ${ }^{1}$ The Food and Drug Administration has given the matter extra attention and taken further precautions to prevent ADRs triggered by dental medications. Hence, dentists must be aware of drug usage for certain oral disease conditions, as well as adverse drug reactions (ADR) to the same medicines.

The significance of medication error was first revealed when the Institute of Medicine (US) reported in "To Err is Human; Building a Safer Health System" that yearly medical errors caused between 44,000 and 98,000 fatalities in the United States. ${ }^{2}$ According to Prof. Jha's research, almost
5.2 million medical mistakes occur in India each year. As per the British Medical Journal, India, like any developing nation, is experiencing a high rate of medical mistakes. ${ }^{3}$ The key explanation for this is that the developing nations lack qualified medical professionals to assess medical outcomes. Khan et al., 2015 conducted a survey involving dental professionals and found that an absence of information and difficulties in dental practitioners' attitudes are the root causes of underreporting of ADRs. Poor understanding of the ADR reporting process, inadequate experience to identify ADRs, psychological concerns, sloth, and a lack of professional responsibility are among the reported issues. ${ }^{4}$

In their usual medical practice, dentists recommend a broad range of drugs to treat of common oral disorders, including antibiotics, analgesics, anti-inflammatory agents, and antipyretics. Dentists are frequently acquainted with adverse drug responses produced by pharmaceuticals that they prescribe for the

(C)2020. Open access. This article is distributed under the terms of the Creative Commons Attribution-Noncommercial-Share Alike 4.0 Unported License. 
management of mucosal disorders in their day-today practice, but they must also be aware of other drugs that might induce oral reactions which necessitate dental intervention.

Pharmacovigilance is a significant tool in the health-care profession that aids in the evaluation, surveillance, and identification of innovative medications for patient benefit. Dental pharmacovigilance is a new and essential trend for dentists in addressing patients who are experiencing adverse side effects from dental drugs.

Drug safety is mostly determined by how drugs are monitored after they have been launched and recommended. To avoid catastrophic drug disasters, it is critical to stimulate a system of safe drug consumption and quick reporting in effort to allow medications more efficient in dental operations. Rare but major adverse events, medication toxicity over time, usage of certain pharmaceuticals in certain populations such as children, pregnant women, or the elderly, and drug interactions are frequently inadequate or not recorded. A lack of attention in the slow reporting of adverse drug reactions might be attributable to a lack of depth of understanding on the part of dentists.

According to Jadhav et al., 2017, 106 dentists in their research had a poor understanding of pharmacovigilance. Perhaps they have a positive attitude about ADR reporting, however, because to a lack of understanding, a lack of ADR reporting training, and a lack of payment for reporting, it is difficult to determine whether or not an ADR has happened. 5 The another problem of pharmacovigilance is that drug firms continue to invest enormous sums of money on promotional releases rather than devoting a percentage of their resources to spreading training and awareness initiatives that encourage early reporting of ADRs. ${ }^{6}$ Hence, dentists must be educated in pharmacovigilance programs. Increased and updated pharmacovigilance knowledge in dental therapies requires proactive engagement from health care providers, particularly dentists.
The complete pharmacovigilance procedure in dentistry begins with the collection of adverse event data from patients. Manufacturers should also assess the severity, expectedness, and causation of data obtained from patients using various methods. It is critical for the dentist, clinical pharmacist, pathologist, or microbiological to provide information on adverse drug reactions. As members of a medication safety team, these professionals can help to promote a culture of safe drug use by reporting adverse drug reactions.

Requested information from patient care platforms, information from clinical trials, postmarketing surveillance reports, reviews from healthcare professionals or patients or other intermediaries, reports from existing literature (reporting is a regulatory healthcare requirement in many countries), reports from the media, and reports reported to drug regulatory authorities themselves form the foundation of adverse drug reaction reports. For creating and executing a thorough pharmaco-vigilance program at a national level, a strong regulatory framework with robust organizational support is critical. By forming a medical safety team at the institutional level, dental schools may collaborate and incorporate organizational approaches to patient safety.

Using relevant evidence-based literature and dental experiences, definitive reports that reflect the patient's reaction can be examined. For gathering information on adverse drug reactions, national pharmacovigilance centers and drug information centers are the most valuable tools. Dentists must recognize that reporting adverse drug reactions promotes the profession of dentistry as a whole. Early and proper documenting of adverse drug reactions will have a direct impact on the speed of preventing ADRs and with which harmful pharmaceuticals may be removed from the market, thereby influencing patients' lives, and the dentistry profession will attain greater safety.

Pharmacovigilance training should be provided to all healthcare professionals, and it should be 
incorporated in undergraduate curriculum to raise awareness among aspiring doctors. The majority of pharmacology instruction in the US medical schools takes place in the first few years of medical school, when students are introduced to fundamental pharmacodynamics and pharmacokinetics concepts, as well as system-based pathophysiology courses. A comprehensive course module in the dentistry curriculum should be incorporated to offer a systematic and broad exposure to the issues of adverse drug events and pharmacovigilance. The curriculum should be specially created to incorporate information throughout dental educational institutions, including concepts of ADR detections, evaluation, reporting, and the relevance of pharmacovigilance with case-based studies.

Dental pharmacovigilance has evolved to the point that dentists must keep up to speed on new pharmaceuticals, drug safety, and treatment trends on a regular basis. While much progress has been made in the field of pharmacovigilance in the West, little has been accomplished in India, where there is an urgent need to bridge the gap between ADR reporting and their prevention.

Conflict of Interest: No relevance of interest declared.

\section{REFERENCES}

1. Sarathy AP, Bourgeois SL Jr, Goodell GG. Bisphosphonate-associated osteonecrosis of the jaws and endodontic treatment: two case reports. J Endod. 2005 Oct;31(10):759-63.

2. Institute of Medicine (US) Committee on Quality of Health Care in America. To Err is Human: Building a Safer Health System. Kohn LT, Corrigan JM, Donaldson MS, editors. Washington (DC): National Academies Press (US); 2000.

3. Zirpe KG, Seta B, Gholap S, Aurangabadi K, Gurav SK, Deshmukh AM, Wankhede P, Suryawanshi P, Vasanth S, Kurian M, Philip E, Jagtap N, Pandit E. Incidence of Medication Error in Critical Care Unit of a Tertiary Care Hospital: Where Do We Stand? Indian J Crit Care Med. 2020 Sep;24(9):799-803.

4. Khan SA, Goyal C, Tonpay SD. A study of knowledge, attitudes, and practice of dental doctors about adverse drug reaction reporting in a teaching hospital in India. Perspect Clin Res. 2015 Jul-Sep;6(3):144-9.

5. Jadhav A, Chandrikapure A, Tarte P. Pharmacovigilance in dental practice: A study to evaluate knowledge, attitude and practices (KAP) of reporting of adverse drug reactions (ADR) among dental practitioner in a city of central region of Maharashtra, India. MedPulse - Int J Dent. April 2017;2(2):14-18.

6. Rodrigues GS, Khan SA. Pharmacovigilance among surgeons and in surgical wards: overlooked or axiomatic? Indian J Surg. 2011 Jan;73(1):4-8.

Cite the Article as: Khalaid M, Khatoon M. Pharmacovigilance Education for Dental Students. J Drug Vigil Altern Ther. 2021 June 30;1(2):89-91.

\section{www.jdvat.org}

(cc) EY-NC-SA This is an open access paper distributed under the copyright agreement with JDVAT, which permits noncommercial unrestricted use, distribution, and reproduction in any medium or format, provided the original work is properly cited. 